\title{
Effect of internal state variables in thermoelasticity of microstretch bodies
}

\author{
Marin Marin and Sorin Vlase
}

\begin{abstract}
First, we formulate the mixed initial boundary value problem in the theory of thermoelastic microstretch bodies having certain internal state variables. Then by using some approachable computing techniques and the known Gronwall's inequality we will prove that the presence of internal state variables do not influence the uniqueness of solution of the mixed problem.
\end{abstract}

The purpose of the theory of thermo-microstretch elastic solids is to eliminate discrepancies between classical elasticity and experiments. This is a theory of thermoelasticity with microstructure that include intrinsic rotations and microstructural expansion and contractions.

The classical elasticity failed to present acceptable results when the effects of material microstructure were known to contribute significantly to the body's overall deformations, for example, in the case of granular bodies with large molecules (e.g. polymers), graphite or human bones.

These cases are becoming increasingly important in the design and manufacture of modern day advanced materials, as small-scale effects become paramount in the prediction of the overall mechanical behaviour of these materials.

Key Words: thermoelasticity, microstretch body, internal state variables, uniqueness of solution, Gronwall's inequality.

2010 Mathematics Subject Classification: Primary 35A25, 35G46, 74A60; Secondary $74 \mathrm{H} 25,80 \mathrm{~A} 20$.

Received: 28.06 .2015

Revised: 10.09.2015

Accepted: 25.09.2015 
Other intended applications of this theory are to composite materials reinforced with chopped fibers and various porous materials.

We must outline that the theory of thermo-microstretch elastic solids was first elaborated by Eringen, [4]. This theory can be useful in the applications which deal with porous materials as geological materials, solid packed granular materials and many others.

On the other hand, materials which operate at elevated temperatures will invarianbly be subjected to heat flow at some time during normal use. Such heat flow will involve a non-linear temperature distribution which will inevitable give rise to thermal stresses. For these reasons, the development, design and selection of materials for high temperature applications requires a great deal of care. The role of the pertinent material properties and other variables which can affect the magnitude of thermal stress must be considered.

The main difficulty of the thermo-microstretch materials is the large number of the thermoelastic coefficients and, as such, the problem of their determination in the laboratory. Yet many authors consider that this problem will be solved in future.

Already, in the isotropic case, when strongly decreases the number of coefficients, they are calculated as can be seen in many works due to Eringen [4] or Iesan and Quintanilla [8].

The present paper must be considered as a first step to a better understanding of microstretch and thermal stress in the study of above ennumerated materials.

Some considerations on the propagation of plane waves in a microstretch thermoelastic diffusion solid of infinite extent can be find in the paper [9] The Newton interpolating series proposed in [6] can be used successfully to approximate the solution of boundary value problems. The impact of initial mechanical deformation on the propagation of plane waves in a linear elastic isotropic solid, is shown in [7], while the algorithm proposed in [5] may be useful in addressing the solutions of mixed initial boundary value problem in the context of microstretch bodies.

Different considerations are given to the solution of the mixed problem in thermoelasticity of microstretch bodies as in papers [10]-[15].

Interest to consider the internal state variables as a means to estimate mechanical properties has grown rapidly in recent years.

The theories of internal state variables in different kind of materials represent a material length scale and are quite sufficient for a large number of the solid mechanics applications.

The internal state variables are the smallest possible subset of system variables that can represent the entire state of the system at any given time. The minimum number of state variables required to represent a given system, $n$, is 
usually equal to the order of the system's defining differential equation. If the system is represented in transfer function form, the minimum number of state variables is equal to the order of the transfer function's denominator after it has been reduced to a proper fraction. It is important to understand that converting a state space realization to a transfer function form may lose some internal information about the system, and may provide a description of a system which is stable, when the state-space realization is unstable at certain points.

The theory of bodies with internal state variables has been first formulated for the thermo-viscoelastic materials (see, for instance Chirita [3]). Then the internal state variables has been considered for different kind of materials.

The study [16] of Nachlinger and Nunziato is dedicated to the internal state variables approach of finite deformations without heat conduction in the one-dimensional case.

In the paper [17] the authors describe how the so-called Bammann internal state variable constitutive approach, which has proven highly successful in modelling deformation processes in metals, can be applied with great benefit to silicate rocks and other geological materials in modelling their deformation dynamics. In its essence, the internal state variables theory provides a constitutive framework to account for changing history states that arise from inelastic dissipative microstructural evolution of a polycrystalline solid.

A thermodynamically consistent framework is proposed for modeling the hysteresis of capillarity in partially saturated porous media in the paper [19].

The paper [2] presents the formulation of a constitutive model for amorphous thermoplastics using a thermodynamic approach with physically motivated internal state variables. The formulation follows current internal state variable methodologies used for metals and departs from the spring-dashpot representation generally used to characterize the mechanical behavior of polymers.

Anand and Gurtin develop in the paper [1] a continuum theory for the elastic-viscoplastic deformation of amorphous solids such as polymeric and metallic glasses. Introducing an internal-state variable that represents the local free-volume associated with certain metastable states, the authors are able to capture the highly non-linear stress-strain behavior that precedes the yield-peak and gives rise to post-yield strain softening.

In the study [18], is presented a formulation of state variable based gradient theory to model damage evolution and alleviate numerical instability associated within the post-bifurcation regime. This proposed theory is developed using basic microforce balance laws and appropriate state variables within a consistent thermodynamic framework. The theory provides a strong coupling and consistent framework to prescribe energy storage and dissipation 
associated with internal damage.

\section{Basic equations}

We consider thermoelastic microstretch body with internal state variables which occupy an open region $B$ of three-dimensional Euclidean space $R^{3}$, at time $t=0$, in it's reference configuration.

The boundary of the domain $B$, denoted by $\partial B$, is a closed, bounded and piece-wise smooth surface which allows us the application of the divergence theorem. A fixed system of rectangular Cartesian axes is used and we adopt the Cartesian tensor notations. The points in $B$ are denoted by $\left(x_{i}\right)$ or $(x)$. The variable $t$ is the time and $t \in\left[0, t_{0}\right)$. We shall employ the usual summation over repeated subscripts while subsripts preceded by a comma denote the partial differentiation with respect to the spatial argument. Also, we use a superposed dot to denote the partial differentiation with respect to $t$. The Latin indices are understood to range over the integers $(1,2,3)$, while the Greek subsripts have the range $1,2, \ldots, n$.

In the following we designate by $n_{i}$ the components of the outward unit normal to the surface $\partial B$. The closure of the domain $B$, denoted by $\bar{B}$, means $\bar{B}=B \cup \partial B$.

Also, the spatial argument and the time argument of a function will be ommited when there is no likelihood of confusion.

The behaviour of a thermoelastic microstretch body is characterized by the following kinematic variables:

$$
u_{i}=u_{i}(x, t), \varphi_{i}=\varphi_{i}(x, t),(x, t) \in B \times\left[0, t_{0}\right)
$$

where $u_{i}$ are the components of the displacement field and $\varphi_{i}$ - the components of the microrotation vector.

The fundamental system of field equations, in the theory of microstretch thermoelastic bodies with internal state variables, consists of:

- the equations of motion:

$$
\begin{gathered}
t_{i j, j}+\varrho F_{i}=\varrho \ddot{u}_{i}, \\
m_{j i, j}+\varepsilon_{i j k} t_{j k}+\varrho G_{i}=I_{i j} \ddot{\varphi}_{j} ;
\end{gathered}
$$

- the energy equation:

$$
T_{0} \dot{\eta}=q_{i, i}+\varrho r ;
$$


- the constitutive equations:

$$
\begin{aligned}
& t_{i j}=A_{i j m n} \varepsilon_{m n}+B_{m n i j} \gamma_{m n}-A_{i j} \theta+a_{i j \alpha} \omega_{\alpha}, \\
& m_{i j}=B_{i j m n} \varepsilon_{m n}+C_{i j m n} \gamma_{m n}-B_{i j} \theta+b_{i j \alpha} \omega_{\alpha}, \\
& \eta=A_{i j} \varepsilon_{i j}+B_{i j} \gamma_{i j}-a \theta-g_{\alpha} \omega_{\alpha}, \\
& q_{i}=C_{i j k} \varepsilon_{j k}+D_{i j k} \gamma_{j k}+d_{i} \theta+f_{i \alpha} \omega_{\alpha}+K_{i j} \theta_{, j} ;
\end{aligned}
$$

- the geometric equations:

$$
\varepsilon_{i j}=u_{j, i}+\varepsilon_{i j k} \varphi_{k}, \quad \gamma_{i j}=\varphi_{j, i}
$$

The internal state variables are denoted by $\xi_{\alpha}, \alpha=1,2, \ldots, n$, but in the linear theory, we denote by $\omega_{\alpha}$ the internal state variables measured from the internal state variables $\xi_{\alpha}^{0}$ of the initial state. Also, the temperature $\theta$ represents the difference between the absolute temperature $T$ and the temperature $T_{0}, T_{0}>0$, of the initial state. Thus we have:

$$
\xi_{\alpha}=\xi_{\alpha}^{0}+\omega_{\alpha}, T=T_{0}+\theta .
$$

Within the linear approximation, from the entropy production inequality, it follows (see, for instance, [1]):

$$
\dot{\omega}_{\alpha}=f_{\alpha},
$$

where

$$
f_{\alpha}=g_{i j \alpha} \varepsilon_{i j}+h_{i j \alpha} \gamma_{i j}+p_{\alpha} \theta+q_{\alpha \beta} \omega_{\beta}+r_{i \alpha} \theta,{ }_{i} .
$$

In the above relations we have used the following notations:

- $\varrho$ - the constant mass density;

- $t_{i j}, m a_{i j}$ - the components of the stress tensors;

- $I_{i j}$ - the coefficients of inertia;

- $F_{i}$ - the components of body force per unit mass;

- $G_{i}$ - the components of body couple per unit mass;

- $r$ - the heat supply per unit mass and unit time;

- $\eta$ - the entropy per unit mass;

- $q_{i}$ - the components of the heat flux;

- $\varepsilon_{i j}, \gamma_{i j}$ - the kinematic characteristics of the strain tensors.

The above coefficients $A_{i j m n}, B_{i j m n}, \ldots, A_{i j}, \ldots, C_{i j k}, \ldots, g_{i j \alpha}, \ldots, r_{i \alpha}$ are functions of $x$ and characterize the thermoelastic properties of the material with internal state variable (the constitutive coefficients). $K_{i j}$ is the conductivity tensor. 
In the case of a homogeneous medium these quantities are constants. The constitutive coefficients obey to the following symmetry relations

$$
\begin{gathered}
A_{i j m n}=A_{m n i j}, B_{i j m n}=B_{m n i j}, A_{i j}=A_{j i}, \\
C_{i j m n}=C_{m n i j}, a_{i j \alpha}=a_{j i \alpha}, K_{i j}=K_{j i} .
\end{gathered}
$$

We supplement the above equations with the following initial conditions

$$
\begin{aligned}
& u_{i}\left(x_{s}, 0\right)=u_{i}^{0}\left(x_{s}\right), \dot{u}_{i}\left(x_{s}, 0\right)=u_{i}^{1}\left(x_{s}\right), \\
& \varphi_{i}\left(x_{s}, 0\right)=\varphi_{i}^{0}\left(x_{s}\right), \dot{\varphi}_{i}\left(x_{s}, 0\right)=\varphi_{i}^{1}\left(x_{s}\right), \\
& \theta\left(x_{s}, 0\right)=\theta^{0}\left(x_{s}\right), \omega_{\alpha}\left(x_{s}, 0\right)=\omega_{\alpha}^{0}\left(x_{s}\right),\left(x_{s}\right) \in B
\end{aligned}
$$

and the prescribed boundary conditions

$$
\begin{aligned}
& u_{i}=\tilde{u}_{i}, \text { on } \overline{\partial B_{1}} \times\left[0, t_{0}\right], t_{i} \equiv t_{i j} n_{j}=\tilde{t_{i}}, \text { on } \partial B_{1}^{c} \times\left[0, t_{0}\right], \\
& \varphi_{i}=\tilde{\varphi}_{i}, \text { on } \overline{\partial B_{2}} \times\left[0, t_{0}\right], m_{i} \equiv m_{i j} n_{j}=\tilde{m}_{i}, \text { on } \partial B_{2}^{c} \times\left[0, t_{0}\right], \\
& \theta=\tilde{\theta}, \text { on } \overline{\partial B_{3}} \times\left[0, t_{0}\right], q \equiv q_{i} n_{i}=\tilde{q}, \text { on } \partial B_{3}^{c} \times\left[0, t_{0}\right] .
\end{aligned}
$$

In (10) the surfaces $\overline{\partial B_{1}}, \overline{\partial B_{2}}, \overline{\partial B_{3}}$ and respective complements $\partial B_{1}^{c}, \partial B_{2}^{c}$, $\partial B_{3}^{c}$ are subsets of the boundary $\partial B$ which satisfay the relations

$$
\begin{aligned}
& \overline{\partial B_{1}} \cup \partial B_{1}^{c}=\overline{\partial B_{2}} \cup \partial B_{2}^{c}=\overline{\partial B_{3}} \cup \partial B_{3}^{c}=\partial B \\
& \partial B_{1} \cap \partial B_{=}^{c} \partial B_{2} \cap \partial B_{2}^{c}=\partial B_{3} \cap \partial B_{3}^{c}=\emptyset
\end{aligned}
$$

The functions $u_{i}^{0}, u_{i}^{1}, \varphi_{i}^{0}, \varphi_{i}^{1}, \theta^{0} \omega_{\alpha}^{0}, \tilde{u}_{i}, \tilde{t}_{i}, \tilde{\varphi}_{i}, \tilde{m}_{i}, \tilde{\theta}$ and $\tilde{q}$, used in the above conditions (9) and (10), are assumed be prescribed in their domain of definition.

In conclusion, the mixed initial boundary value problem of the thermoelasticity of microstretch bodies with internal variables consists of the equations $(1),(2)$ and (6), the initial conditions (9) and the boundary conditions (10).

By a solution of this problem we mean an ordered array $\left(u_{i}, \varphi_{i}, \theta, \omega_{\alpha}\right)$ satisfying the Eqns. (1), (2) and (6) and the conditions (9) and (10).

\section{Main results}

In this section we shall deduce some estimations and then, as a conse-

quence, we obtain in simple manner the uniqueness theorem of the solution of the above problem.

In order to prove these results, we shall need the following assumptions

- (i) the mass density $\varrho$ is strictly positive, i.e.

$$
\varrho\left(x_{s}\right) \geq \varrho_{0}>0, \text { on } B
$$


- (ii) there exists a positive constant $\lambda_{1}$ such that

$$
I_{i j} \xi_{i} \xi_{j} \geq \lambda_{1} \xi_{i} \xi_{i}, \forall \xi_{i}
$$

- (iii) the specific heat $a$ from $(3)_{4}$ is strictly positive, i.e.

$$
a\left(x_{s}\right) \geq a_{0}>0, \text { on } B
$$

- (iv) the constitutive tensors $A_{i j m n}, B_{i j m n}$ and $C_{i j m n}$ are positive definite, in the following sense:

$$
\begin{aligned}
& \int_{B} A_{i j m n} \xi_{i j} \xi_{m n} d v \geq \lambda_{2} \int_{B} \xi_{i j} \xi_{i j} d v, \forall \xi_{i j} \\
& \int_{B} B_{i j m n} \xi_{i j} \xi_{m n} d v \geq \lambda_{3} \int_{B} \xi_{i j} \xi_{i j} d v, \forall \xi_{i j} \\
& \int_{B} C_{i j m n} \xi_{i j} \xi_{m n} d v \geq \lambda_{4} \int_{B} \xi_{i j} \xi_{i j} d v, \forall \xi_{i j}
\end{aligned}
$$

where $\lambda_{2}, \lambda_{3}$ and $\lambda_{4}$ are positive constants;

- (v) the symmetric part $\tilde{K}_{i j}$ of the thermal conductivity tensor $K_{i j}$ is positive definite, in the sense that there exists a positive constant $\mu$ such that

$$
\int_{B} \tilde{K}_{i j} \xi_{i} \xi_{j} d v \geq \mu \int_{B} \xi_{i} \xi_{i} d v, \text { for all vectors } \xi_{i} .
$$

Consider that our mixed problem has two solutions

$$
\left(u_{i}^{(\nu)}, \varphi_{i}^{(\nu)}, \theta^{(\nu)}, \omega_{\alpha}^{(\nu)}\right), \nu=1,2
$$

two solutions of our initial boundary value problem.

Because of the linearity of the problem, their difference is also solution of the problem. We denote by $\left(v_{i}, \psi_{i}, \vartheta, w_{\alpha}\right)$ the differences,

$$
v_{i}=u_{i}^{(2)}-u_{i}^{(1)}, \psi_{i}=\varphi_{i}^{(2)}-\varphi_{i}^{(1)}, \vartheta=\theta^{(2)}-\theta^{(1)}, w_{\alpha}=\omega_{\alpha}^{(2)}-\omega_{\alpha}^{(1)}
$$

In order to prove the desired uniquness theorem, it suffice to prove that the above considered problem, consists of the equations (1), (2) and (6) and the conditions (9) and (10), in which

$$
\begin{aligned}
& F_{i}=G_{i}=r=0 \\
& \quad u_{i}^{0}=u_{i}^{1}=\varphi_{i}^{0}=\varphi_{i}^{1}=\theta^{0}=\omega_{\alpha}^{0}=0
\end{aligned}
$$

and

$$
\tilde{u}_{i}=\tilde{t}_{i}=\tilde{\varphi}_{i}=\tilde{m}_{i j}=\tilde{\theta}=\tilde{q}=0
$$


imply that

$$
v_{i}=\psi_{i}=\vartheta=w_{\alpha}=0,
$$

in $B \times\left[0, t_{0}\right]$, provided that the hypotheses (i) - (v) hold.

So, we have a new problem, let say $P_{0}$, defined by the following equations

$$
\begin{gathered}
t_{i j, j}=\varrho \ddot{u}_{i}, \\
m_{i j, j}+\varepsilon_{i j k} t_{j k}=I_{i j} \ddot{\varphi}_{j} \\
T_{0} \dot{\eta}=q_{i, i} \\
\dot{\omega}_{\alpha}=f_{\alpha},
\end{gathered}
$$

with the initial conditions

$$
\begin{aligned}
& u_{i}\left(x_{s}, 0\right)=0, \dot{u}_{i}\left(x_{s}, 0\right)=0, \varphi_{i}\left(x_{s}, 0\right)=0, \\
& \dot{\varphi}_{i}\left(x_{s}, 0\right)=0, \theta\left(x_{s}, 0\right)=0, \omega_{\alpha}\left(x_{s}, 0\right)=0,\left(x_{s}\right) \in B
\end{aligned}
$$

and the boundary conditions

$$
\begin{aligned}
& u_{i}=0, \text { on } \overline{\partial B_{1}} \times\left[0, t_{0}\right], t_{i} \equiv t_{i j} n_{j}=0, \text { on } \partial B_{1}^{c} \times\left[0, t_{0}\right], \\
& \varphi_{i}=0, \text { on } \overline{\partial B_{2}} \times\left[0, t_{0}\right], m_{i} \equiv m_{i j} n_{j}=0, \text { on } \partial B_{2}^{c} \times\left[0, t_{0}\right], \\
& \theta=0, \text { on } \overline{\partial B_{3}} \times\left[0, t_{0}\right], q \equiv q_{i} n_{i}=0, \text { on } \partial B_{3}^{c} \times\left[0, t_{0}\right]
\end{aligned}
$$

Together with these equations and conditions we take into account the constitutive relations (3) and (7). In order to prove that the problem $P_{0}$ admits the null solution, we will show that the function $y(t)$ defined by

$$
y(t)=\int_{B}\left(\dot{u}_{i} \dot{u}_{i}+\dot{\varphi}_{i} \dot{\varphi}_{i}+\varepsilon_{i j} \varepsilon_{i j}+\gamma_{i j} \gamma_{i j}+\theta^{2}+\omega_{\alpha} \omega_{\alpha}\right) d V
$$

vanishes on $\left[0, t_{0}\right]$.

To this aim, we first prove some useful estimations.

Theorem 1. If the ordered array $\left(u_{i}, \varphi_{i}, \theta, \omega_{\alpha}\right)$ is a solution of the problem $P_{0}$, then the following relation holds

$$
\begin{aligned}
\frac{1}{2} \int_{B}\left(A_{i j m n} \varepsilon_{i j} \varepsilon_{m n}+2 B_{i j m n} \varepsilon_{i j} \gamma_{m n}+B_{i j m n} \gamma_{i j} \gamma_{m n}+\right. \\
\left.+2 a_{i j \alpha} \varepsilon_{i j} \omega_{\alpha}+2 b_{i j \alpha} \gamma_{i j} \omega_{\alpha}+a \theta^{2}+\varrho \dot{u}_{i} \dot{u}_{i}+I_{i j} \dot{\varphi}_{i} \dot{\varphi}_{j}\right) d V= \\
=\int_{0}^{t} \int_{B}\left(a_{i j \alpha} \varepsilon_{i j} \dot{\omega}_{\alpha}+b_{i j \alpha} \gamma_{i j} \dot{\omega}_{\alpha}+g_{\alpha} \theta \dot{\omega}_{\alpha}-\frac{1}{T_{0}} q_{i} \theta, i\right] d V d s
\end{aligned}
$$


Proof. In view of constitutive equations (3) and the symmetry relations (8), we obtain

$$
\begin{aligned}
& t_{i j} \dot{\varepsilon}_{i j}+ m_{i j} \dot{\gamma}_{i j}+\dot{\eta} \theta= \\
&+ A_{i j m n} \dot{\varepsilon}_{i j} \varepsilon_{m n}+B_{m n i j} \varepsilon_{m n} \dot{\varepsilon}_{i j}-A_{i j} \dot{\varepsilon}_{i j} \theta+a_{i j \alpha} \dot{\varepsilon}_{i j} \omega_{\alpha}+ \\
& \quad B_{i j m n} \varepsilon_{m n} \dot{\gamma}_{i j}+C_{m n i j} \gamma_{m n} \dot{\gamma}_{i j}-B_{i j} \dot{\gamma}_{i j} \theta+b_{i j \alpha} \dot{\gamma}_{i j} \omega_{\alpha}+ \\
& \quad+A_{i j} \dot{\varepsilon}_{i j} \theta+B_{i j} \dot{\gamma}_{i j} \theta+a \dot{\theta} \theta-g_{\alpha} \dot{\omega}_{\alpha} \theta= \\
&=\frac{1}{2} \frac{\partial}{\partial t}\left(A_{i j m n} \varepsilon_{i j} \varepsilon_{m n}+2 B_{m n i j} \varepsilon_{i j} \gamma_{m n}+C_{i j m n} \gamma_{i j} \gamma_{m n}+a \theta^{2}+\right. \\
&\left.\quad+2 a_{i j \alpha} \varepsilon_{i j} \omega_{\alpha}+2 b_{i j \alpha} \gamma_{i j} \omega_{\alpha}\right)-a_{i j \alpha} \varepsilon_{i j} \dot{\omega}_{\alpha}-b_{i j \alpha} \gamma_{i j} \dot{\omega}_{\alpha}-g_{\alpha} \theta \dot{\omega}_{\alpha} .
\end{aligned}
$$

On the other hand, in view of equations (11), (12) and the geometric relations (4), we are lead to the relation

$$
\begin{aligned}
& t_{i j} \dot{\varepsilon}_{i j}+m_{i j} \dot{\gamma}_{i j}+\dot{\eta} \theta=t_{i j}\left(\dot{u}_{j, i}+\varepsilon_{i j k} \dot{\varphi}_{k}\right)+m_{i j} \dot{\varphi}_{j, i}+\frac{1}{T_{0}} q_{i, i} \theta= \\
& =\left(t_{i j} \dot{u}_{j}\right)_{, i}-t_{i j, i} \dot{u}_{j}+\varepsilon_{i j k} \dot{\varphi}_{k} t_{i j}+\left(m_{i j} \dot{\varphi}_{j}\right)_{, i}-m_{i j, i} \dot{\varphi}_{j}+\left(\frac{1}{T_{0}} q_{i} \theta\right)_{, i}-\frac{1}{T_{0}} q_{i} \theta,_{i} \\
& =\left(t_{i j} \dot{u}_{j}+m_{i j} \dot{\varphi}_{j}+\frac{1}{T_{0}} q_{i} \theta\right)_{, i}-\frac{1}{2} \frac{\partial}{\partial t}\left(\varrho \dot{u}_{i} \dot{u}_{i}+I_{i j} \dot{\varphi}_{i} \dot{\varphi}_{j}\right)-\frac{1}{T_{0}} q_{i} \theta_{, i}
\end{aligned}
$$

From the equalities (17) and (18) we obtain

$$
\begin{gathered}
\frac{1}{2} \frac{\partial}{\partial t}\left(A_{i j m n} \varepsilon_{i j} \varepsilon_{m n}+2 B_{m n i j} \varepsilon_{i j} \gamma_{m n}+C_{i j m n} \gamma_{i j} \gamma_{m n}+\right. \\
\left.+2 a_{i j \alpha} \varepsilon_{i j} \omega_{\alpha}+2 b_{i j \alpha} \gamma_{i j} \omega_{\alpha}+a \theta^{2}+\varrho \dot{u}_{i} \dot{u}_{i}+I_{i j} \dot{\varphi}_{i} \dot{\varphi}_{j}\right)= \\
=\left(t_{i j} \dot{u}_{j}+m_{i j} \dot{\varphi}_{j}+\frac{1}{T_{0}} q_{i} \theta\right)_{, i}-\frac{1}{T_{0}} q_{i} \theta{ }_{, i}+ \\
+a_{i j \alpha} \varepsilon_{i j} \dot{\omega}_{\alpha}+b_{i j \alpha} \gamma_{i j} \dot{\omega}_{\alpha}+g_{\alpha} \theta \dot{\omega}_{\alpha}
\end{gathered}
$$

Now, we integrate relation (19) over the domain $B$. By using the divergence theorem and the boundary conditions (15), we conclude that

$$
\begin{array}{r}
\frac{1}{2} \frac{\partial}{\partial t} \int_{B}\left(A_{i j m n} \varepsilon_{i j} \varepsilon_{m n}+2 B_{m n i j} \varepsilon_{i j} \gamma_{m n}+C_{i j m n} \gamma_{i j} \gamma_{m n}+\right. \\
\left.+2 a_{i j \alpha} \varepsilon_{i j} \omega_{\alpha}+2 b_{i j \alpha} \gamma_{i j} \omega_{\alpha}+a \theta^{2}+\varrho \dot{u}_{i} \dot{u}_{i}+I_{i j} \dot{\varphi}_{i} \dot{\varphi}_{j}\right) d V= \\
=\int_{B}\left(a_{i j \alpha} \varepsilon_{i j} \dot{\omega}_{\alpha}+b_{i j \alpha} \gamma_{i j} \dot{\omega}_{\alpha}+g_{\alpha} \theta \dot{\omega}_{\alpha}-\frac{1}{T_{0}} q_{i} \theta, i\right) d V .
\end{array}
$$

Finally, we integrate the equality $(20)$ on $[0, t]$ and, by using the null initial condition (14), we arrive at the desired result (16). 
Theorem 2. Let $\left(u_{i}, \varphi_{i}, \theta, \omega_{\alpha}\right)$ be a solution of the problem $P_{0}$. Then there exists the positive constants $m_{1}$ and $m_{2}$ such that the following relation hold

$$
\begin{aligned}
& \int_{B}\left[\left(a_{i j \alpha} \varepsilon_{i j}+b_{i j \alpha} \gamma_{i j}+g_{\alpha} \theta\right) \dot{\omega}_{\alpha}-\frac{1}{T_{0}} q_{i} \theta, i\right] d V \leq \\
& \leq-m_{1} \int_{B} \theta,{ }_{i} \theta,{ }_{j} d V+m_{2} \int_{B}\left(\varepsilon_{i j} \varepsilon_{i j}+\gamma_{i j} \gamma_{i j}+\theta^{2}+\omega_{\alpha} \omega_{\alpha}\right) d V .
\end{aligned}
$$

Proof. Taking into account the relations $(6),(7)$ and $(3)_{4}$, we can write:

$$
\begin{gathered}
a_{i j \alpha} \varepsilon_{i j} \dot{\omega}_{\alpha}+b_{i j \alpha} \gamma_{i j} \dot{\omega}_{\alpha}+g_{\alpha} \theta \dot{\omega}_{\alpha}-\frac{1}{T_{0}} q_{i} \theta, i= \\
=\left(a_{i j \alpha} \varepsilon_{i j}+b_{i j \alpha} \gamma_{i j}+g_{\alpha} \theta\right)\left(g_{m n \alpha} \varepsilon_{m n}+h_{m n \alpha} \gamma_{m n}+p_{\alpha} \theta+q_{\alpha \beta} \omega_{\beta}+r_{k \alpha} \theta, k\right) \\
\quad-\frac{1}{T_{0}}\left(C_{k i j} \varepsilon_{i j}+D_{k i j} \gamma_{i j}+d_{k} \theta+f_{k \alpha} \omega_{\alpha}+K_{k j} \theta, j\right) \theta, k= \\
=\frac{1}{2}\left(a_{i j \alpha} g_{m n \alpha}+a_{m n \alpha} g_{i j \alpha}\right) \varepsilon_{i j} \varepsilon_{m n}+\left(a_{i j \alpha} h_{m n \alpha}+b_{m n \alpha} g_{i j \alpha}\right) \varepsilon_{i j} \gamma_{m n} \\
+\frac{1}{2}\left(b_{i j \alpha} h_{m n \alpha}+b_{m n \alpha} h_{i j \alpha}\right) \gamma_{i j} \gamma_{m n}+\left(a_{i j \alpha} p_{\alpha}+g_{i j \alpha} g_{\alpha}\right) \varepsilon_{i j} \theta+a_{i j \alpha} q_{\alpha \beta} \varepsilon_{i j} \omega_{\beta} \\
+\left(b_{i j \alpha} p_{\alpha}+h_{i j \alpha} g_{\alpha}\right) \gamma_{i j} \theta+\left(a_{i j \alpha} r_{k \alpha}-\frac{1}{T_{0}} C_{k i j}\right) \varepsilon_{i j} \theta,{ }_{k}+g_{\beta} q_{\beta \alpha} \theta \omega_{\alpha}+ \\
+b_{i j \alpha} q_{\alpha \beta} \gamma_{i j} \omega_{\beta}+\left(b_{i j \alpha} r_{k \alpha}-\frac{1}{T_{0}} D_{k i j}\right) \gamma_{i j} \theta, k+g_{\alpha} p_{\alpha} \theta^{2}+ \\
+\left(g_{\alpha} r_{i \alpha}-\frac{1}{T_{0}} d_{i}\right) \theta \theta, i-\frac{1}{T_{0}} f_{i \alpha} \omega_{\alpha} \theta, i-\frac{1}{T_{0}} K_{i j} \theta_{, i} \theta, j
\end{gathered}
$$

For the sake of simplicity we introduce the following notations

$$
\begin{aligned}
& \mathcal{A}_{i j m n}=\frac{1}{2}\left(a_{i j \alpha} g_{m n \alpha}+a_{m n \alpha} g_{i j \alpha}\right), \mathcal{B}_{i j m n}=a_{i j \alpha} h_{m n \alpha}+b_{m n \alpha} g_{i j \alpha}, \\
& \mathcal{C}_{i j m n}=\frac{1}{2}\left(b_{i j \alpha} h_{m n \alpha}+b_{m n \alpha} h_{i j \alpha}\right), \mathcal{D}_{i j}=a_{i j \alpha} p_{\alpha}+g_{i j \alpha} g_{\alpha}, \\
& \mathcal{E}_{i j}=b_{i j \alpha} p_{\alpha}+h_{i j \alpha} g_{\alpha}, \mathcal{B}_{i j \alpha}=a_{i j \beta} q_{\beta \alpha}, \mathcal{D}_{i j \alpha}=b_{i j \beta} q_{\beta \alpha}, \\
& \mathcal{A}_{i j k}=a_{i j \alpha} r_{k \alpha}-\frac{1}{T_{0}} C_{k i j}, \mathcal{B}_{i j k}=b_{i j \alpha} r_{k \alpha}-\frac{1}{T_{0}} D_{k i j}, \mathcal{M}=g_{\alpha} p_{\alpha}, \\
& \mathcal{L}_{\alpha}=G_{\beta} q_{\beta \alpha}, \mathcal{D}_{i}=g_{\alpha} r_{i \alpha}-\frac{1}{T_{0}} d_{i}, \mathcal{F}_{i \alpha}=-\frac{1}{T_{0}} f_{i \alpha}
\end{aligned}
$$

Now, we introduce (23) into (22), then we integrate over $B$ so that we are lead 
to

$$
\begin{aligned}
& \int_{B}\left[\left(a_{i j \alpha} \varepsilon_{i j}+b_{i j \alpha} \gamma_{i j}+g_{\alpha} \theta\right) \dot{\omega}_{\alpha}-\frac{1}{T_{0}} q_{i} \theta, i d V=\right. \\
& =\int_{B}\left(\mathcal{A}_{i j m n} \varepsilon_{i j} \varepsilon_{m n}+\mathcal{B}_{i j m n} \varepsilon_{i j} \gamma_{m n}+\mathcal{C}_{i j m n} \gamma_{i j} \gamma_{m n}+\mathcal{D}_{i j} \varepsilon_{i j} \theta+\right. \\
& \quad+\mathcal{E}_{i j} \gamma_{i j} \theta+\mathcal{B}_{i j \alpha} \varepsilon_{i j} \omega_{\alpha}+\mathcal{D}_{i j \alpha} \gamma_{i j} \omega_{\alpha}+\mathcal{A}_{i j k} \varepsilon_{i j} \theta_{, k}+\mathcal{B}_{i j k} \gamma_{i j} \theta_{, k}+ \\
& \left.\quad+\mathcal{M} \theta^{2}+\mathcal{L}_{\alpha} \theta \omega_{\alpha}+\mathcal{D}_{i} \theta \theta,{ }_{i}+\mathcal{F}_{i \alpha} \omega_{\alpha} \theta, i\right) d V-\int_{B} \frac{1}{T_{0}} K_{i j} \theta_{, i} \theta_{, j} d V .
\end{aligned}
$$

On the terms in the right hand side of (24) we will use the Schwarz's inequality and the arithmetic - geometric mean inequality

$$
a b \leq \frac{1}{2}\left(\frac{a^{2}}{p^{2}}+b^{2} p^{2}\right)
$$

through a convenient choice of the parameter $p$.

For instance, we can write

$$
2 \int_{B} \mathcal{A}_{i j k} \varepsilon_{i j} \theta,{ }_{k} d V \leq p_{1}^{2} \int_{B} \theta_{, i} \theta,{ }_{j} d V+\frac{M_{1}^{2}}{p_{1}^{2}} \int_{B} \varepsilon_{i j} \varepsilon_{i j} d V
$$

and so on.

Thus we have, for arbitrary positive constants $p_{1}, p_{2}, p_{3}$ and $p_{4}$

$$
\begin{gathered}
\int_{B} \int_{B}\left[\left(a_{i j \alpha} \varepsilon_{i j}+b_{i j \alpha} \gamma_{i j}+g_{\alpha} \theta\right) \dot{\omega}_{\alpha}-\frac{1}{T_{0}} q_{i} \theta, i\right] d V \leq \\
\leq\left(-2 \mu+p_{1}^{2}+p_{2}^{2}+p_{3}^{2}+p_{4}^{2}\right) \int_{B} \theta,{ }_{i} \theta,{ }_{i} d V+ \\
+\left(\frac{M_{1}^{2}}{p_{1}^{2}}+M_{5}^{2}+M_{7}^{2}+M_{8}^{2}+M_{10}^{2}\right) \int_{B} \varepsilon_{i j} \varepsilon_{i j} d V+ \\
\quad+\left(\frac{M_{2}^{2}}{p_{2}^{2}}+M_{6}^{2}+M_{9}^{2}+M_{11}^{2}+1\right) \int_{B} \gamma_{i j} \gamma_{i j} d V+ \\
+\left(\frac{M_{3}^{2}}{p_{3}^{2}}+M_{12}^{2}+3\right) \int_{B} \theta^{2} d V+\left(\frac{M_{4}^{2}}{p_{4}^{2}}+M_{13}^{2}+2\right) \int_{B} \omega_{\alpha} \omega_{\alpha} d V
\end{gathered}
$$

where $p_{1}, p_{2}, p_{3}$ and $p_{4}$ are arbitrary positive constants. Also, in the inequality (26) we have used the notations

$$
\begin{gathered}
M_{1}^{2}=\max \left(\mathcal{A}_{i j k} \mathcal{A}_{i j k}\right)\left(x_{s}\right), M_{2}^{2}=\max \left(\mathcal{B}_{i j k} \mathcal{B}_{i j k}\right)\left(x_{s}\right), M_{3}^{2}=\max \left(\mathcal{D}_{i \alpha} \mathcal{D}_{i \alpha}\right)\left(x_{s}\right), \\
M_{4}^{2}=\max \left(\mathcal{F}_{i \alpha} \mathcal{F}_{i \alpha}\right)\left(x_{s}\right), M_{5}^{2}=2 \max \left[\left(\mathcal{A}_{i j m n} \mathcal{A}_{i j m n}\right)\left(x_{s}\right)\right]^{1 / 2}, \\
M_{6}^{2}=2 \max \left[\left(\mathcal{C}_{i j m n} \mathcal{C}_{i j m n}\right)\left(x_{s}\right)\right]^{1 / 2}, M_{7}^{2}=\max \left(\mathcal{B}_{i j m n} \mathcal{B}_{i j m n}\right)\left(x_{s}\right), \\
M_{8}^{2}=\max \left(\mathcal{D}_{i j} \mathcal{D}_{i j}\right)\left(x_{s}\right), M_{9}^{2}=\max \left(\mathcal{E}_{i j} \mathcal{E}_{i j}\right)\left(x_{s}\right), M_{10}^{2}=\max \left(\mathcal{B}_{i j \alpha} \mathcal{B}_{i j \alpha}\right)\left(x_{s}\right), \\
M_{11}^{2}=\max \left(\mathcal{D}_{i j \alpha} \mathcal{D}_{i j \alpha}\right)\left(x_{s}\right), M_{12}^{2}=2 \max \left|\mathcal{M}\left(x_{s}\right)\right|, M_{13}^{2}=\max \left(\mathcal{L}_{\alpha} \mathcal{L}_{\alpha}\right)\left(x_{s}\right),
\end{gathered}
$$


Since the parameters $p_{1}, p_{2}, p_{3}$ and $p_{4}$ are arbitrary, we can choose them such that the number $m_{1}$ defined by

$$
m_{1}=\mu-\frac{1}{2}\left(p_{1}^{2}+p_{2}^{2}+p_{3}^{2}+p_{4}^{2}\right)
$$

is strictly positive. Also, if we choose the constant $m_{2}$ as follows

$$
\begin{aligned}
& m_{2}=\frac{1}{2} \max \left\{\frac{M_{1}^{2}}{p_{1}^{2}}+M_{5}^{2}+M_{7}^{2}+M_{8}^{2}+M_{10}^{2},\right. \\
& \frac{M_{2}^{2}}{p_{2}^{2}}+M_{6}^{2}+M_{9}^{2}+M_{11}^{2}+1, \frac{M_{3}^{2}}{p_{3}^{2}}+M_{12}^{2}+3, \\
&\left.\frac{M_{4}^{2}}{\pi_{4}^{2}}+M_{8}^{2}+M_{18}^{2}+M_{19}^{2}+2, \frac{M_{4}^{2}}{p_{4}^{2}}+M_{13}^{2}+2\right\}
\end{aligned}
$$

then we arrive to the estimate (21) and this conclude the proof of Theorem 2.

Theorem 3. Let $\left(u_{i}, \varphi_{i}, \theta, \omega_{\alpha}\right)$ be a solution of the problem $P_{0}$ and suppose that the assumptions (i) - (v) are satisfied. Then there exists a positive constant $m_{3}$ such that we have the following inequality

$$
\begin{aligned}
& \int_{B}\left(\dot{u}_{i} \dot{u}_{i}+\dot{\varphi}_{i} \dot{\varphi}_{i}+\varepsilon_{i j} \varepsilon_{i j}+\gamma_{i} \gamma_{i}+\theta^{2}+\omega_{\alpha} \omega_{\alpha}\right) d V \leq \\
& \quad \leq m_{3} \int_{0}^{t} \int_{B}\left(\dot{u}_{i} \dot{u}_{i}+\dot{\varphi}_{i} \dot{\varphi}_{i}+\varepsilon_{i j} \varepsilon_{i j}+\gamma_{i j} \gamma_{i j}+\theta^{2}+\omega_{\alpha} \omega_{\alpha}\right) d V d s
\end{aligned}
$$

for any $t \in\left[0, t_{0}\right]$.

Proof. First, taking into account the hypotheses (i) - (v), we have

$$
\begin{aligned}
& m_{0} \int_{B}\left(\dot{u}_{i} \dot{u}_{i}+\dot{\varphi}_{i} \dot{\varphi}_{i}+\varepsilon_{i j} \varepsilon_{i j}+\gamma_{i j} \gamma_{i j}+\theta^{2}\right) d V \leq \\
& \leq \int_{B}\left(A_{i j m n} \varepsilon_{i j} \varepsilon_{m n}+2 B_{i j m n} \varepsilon_{i j} \gamma_{m n}+C_{i j m n} \gamma_{i j} \gamma_{m n}+\right. \\
& \left.\quad+2 a_{i j \alpha} \varepsilon_{i j} \omega_{\alpha}+2 b_{i j \alpha} \gamma_{i j} \omega_{\alpha} a \theta^{2}+\varrho \dot{u}_{i} \dot{u}_{i}+I_{i j} \dot{\varphi}_{i} \dot{\varphi}_{j}\right) d V
\end{aligned}
$$

where we have used the notation

$$
m_{0}=\min \left\{\varrho, a, \lambda_{1}, \lambda_{2}, \lambda_{3}, \lambda_{4}\right\}
$$

Next, we use the Schwarz's inequality and the arithmetic - geometric mean inequality (25) to the right hand side of the relation (28). For arbitrary positive 
constants $p_{5}, p_{6}$ and $p_{7}$, we have

$$
\begin{aligned}
2 \int_{B} B_{i j m n} \varepsilon_{i j} \gamma_{m n} d V & \leq p_{5}^{2} \int_{B} \varepsilon_{i j} \varepsilon_{i j} d V+\frac{N_{1}^{2}}{p_{5}^{2}} \int_{B} \gamma_{i j} \gamma_{i j} d V, \\
2 \int_{B} a_{i j \alpha} \varepsilon_{i j} \omega_{\alpha} d V & \leq p_{6}^{2} \int_{B} \varepsilon_{i j} \varepsilon_{i j} d V+\frac{N_{2}^{2}}{p_{6}^{2}} \int_{B} \omega_{\alpha} \omega_{\alpha} d V \\
2 \int_{B} b_{i j \alpha} \gamma_{i j} \omega_{\alpha} d V & \leq p_{7}^{2} \int_{B} \gamma_{i j} \gamma_{i j} d V+\frac{N_{3}^{2}}{p_{7}^{2}} \int_{B} \omega_{\alpha} \omega_{\alpha} d V,
\end{aligned}
$$

where we have used the notations

$N_{1}^{2}=\max \left(B_{i j m n} B_{i j m n}\right)\left(x_{s}\right), N_{2}^{2}=\max \left(a_{i j \alpha} a_{i j \alpha}\right)\left(x_{s}\right), N_{3}^{2}=\max \left(b_{i j \alpha} b_{i j \alpha}\right)\left(x_{s}\right)$

So, from (16), (21), (28) and (29) we are lead to the inequality

$$
\begin{aligned}
m_{0} \int_{B}\left(\dot{u}_{i} \dot{u}_{i}+\dot{\varphi}_{i} \dot{\varphi}_{i}+\varepsilon_{i j} \varepsilon_{i j}+\gamma_{i j} \gamma_{i j}+\theta^{2}\right) d V \leq \\
\leq\left(p_{5}^{2}+p_{6}^{2}\right) \int_{B} \varepsilon_{i j} \varepsilon_{i j} d V+\left(\frac{N_{1}^{2}}{p_{5}^{2}}+p_{7}^{2}\right) \int_{B} \gamma_{i j} \gamma_{i j} d V+ \\
+\left(\frac{N_{2}^{2}}{p_{6}^{2}}+\frac{N_{3}^{2}}{p_{7}^{2}}\right) \int_{B} \omega_{\alpha} \omega_{\alpha} d V-m_{1} \int_{0}^{t} \int_{B} \theta_{, i} \theta,{ }_{i} d V d s+ \\
\quad+m_{2} \int_{0}^{t} \int_{B}\left(\varepsilon_{i j} \varepsilon_{i j}+\gamma_{i j} \gamma_{i j}+\theta^{2}+\omega_{\alpha} \omega_{\alpha}\right) d V d s
\end{aligned}
$$

where $t \in\left[0, t_{0}\right]$.

Now, by using the null initial conditions (15), the consitutive relation (7) and the equation (13), we arrive to the conclusion that:

$$
\begin{array}{r}
\int_{B} \omega_{\alpha} \omega_{\alpha} d V=\int_{0}^{t} \frac{d}{d s}\left(\int_{B} \omega_{\alpha} \omega_{\alpha} d V\right) d s=2 \int_{0}^{t}\left(\int_{B} \omega_{\alpha} \dot{\omega}_{\alpha} d V\right) d s= \\
=2 \int_{0}^{t} \int_{B}\left(g_{i j \alpha} \varepsilon_{i j}+h_{i j \alpha} \gamma_{i j}+p_{\alpha} \theta+q_{\alpha \beta} \omega_{\beta}+r_{i} \theta, i\right) \omega_{\alpha} d V d s
\end{array}
$$

Now, by using, again, the Schwarz's inequality and the arithmetic - geometric mean inequality (25) to the right hand side of the relation (31). So, we deduce that for an arbitrary positive constant $p_{8}$ the following inequality hold:

$$
\begin{array}{r}
2 \int_{B}\left(g_{i j \alpha} \varepsilon_{i j} \omega_{\alpha}+h_{i j \alpha} \gamma_{i j} \omega_{\alpha}+p_{\alpha} \theta \omega_{\alpha}+q_{\alpha \beta} \omega_{\beta} \omega_{\alpha}+r_{i} \omega_{\alpha} \theta, i\right) d V \leq \\
\leq p_{8}^{2} \int_{B} \theta,{ }_{i} \theta_{, i} d V+\left(\frac{Q_{5}^{2}}{p_{8}^{2}}+Q_{3}^{2}+Q_{4}^{2}+2\right) \int_{B} \omega_{\alpha} \omega_{\alpha} d V+ \\
+Q_{1}^{2} \int_{B} \varepsilon_{i j} \varepsilon_{i j} d V+Q_{2}^{2} \int_{B} \gamma_{i j} \gamma_{i j} d V+\int_{B} \theta^{2} d V
\end{array}
$$


where $t \in\left[0, t_{0}\right]$ and we have used the notations

$$
\begin{gathered}
Q_{1}^{2}=\max \left(g_{i j \alpha} g_{i j \alpha}\right)\left(x_{s}\right), Q_{2}^{2}=\max \left(h_{i j \alpha} h_{i j \alpha}\right)\left(x_{s}\right), Q_{3}^{2}=\max \left(p_{\alpha} p_{\alpha}\right)\left(x_{s}\right), \\
Q_{4}^{2}=2 \max \left[\left(q_{\alpha \beta} q_{\alpha \beta}\right)\left(x_{s}\right)\right]^{1 / 2}, Q_{5}^{2}=\max \left(r_{i \alpha} r_{i \alpha}\right)\left(x_{s}\right),\left(x_{s}\right) \in \bar{B}
\end{gathered}
$$

If we denote by $m_{4}$ the quantity

$$
m_{4}=\max \left\{\frac{Q_{5}^{2}}{p_{8}^{2}}+Q_{3}^{2}+Q_{4}^{2}+2, Q_{1}^{2}, Q_{2}^{2}, 1\right\},
$$

then, from (31) and (32) we obtain the following inequality

$$
\begin{aligned}
\int_{B} \omega_{\alpha} \omega_{\alpha} d V \leq & p_{8}^{2} p_{9}^{2} \int_{0}^{t} \int_{B} \theta_{, i} \theta_{, i} d V d s+ \\
& +m_{4} p_{9}^{2} \int_{0}^{t} \int_{B}\left(\varepsilon_{i j} \varepsilon_{i j}+\gamma_{i j} \gamma_{i j}+\theta^{2}+\omega_{\alpha} \omega_{\alpha}\right) d V d s
\end{aligned}
$$

From (30) and (33) we obtain

$$
\begin{gathered}
m_{0} \int_{B}\left(\dot{u}_{i} \dot{u}_{i}+\dot{\varphi}_{i} \dot{\varphi}_{i}+\theta^{2}\right) d V+\left(m_{0}-p_{5}^{2}-p_{6}^{2}\right) \int_{B} \varepsilon_{i j} \varepsilon_{i j} d V+ \\
+\left(m_{0}-\frac{N_{1}^{2}}{p_{5}^{2}}-p_{7}^{2}\right) \int_{B} \gamma_{i j} \gamma_{i j} d V+\left(p_{9}^{2}-\frac{N_{2}^{2}}{p_{6}^{2}}-\frac{N_{3}^{2}}{p_{7}^{2}}\right) \int_{B} \int_{B} \omega_{\alpha} \omega_{\alpha} d V d V \leq(34) \\
\leq\left(m_{2}+m_{4} p_{9}^{2}\right) \int_{0}^{t} \int_{B}\left(\varepsilon_{i j} \varepsilon_{i j}+\gamma_{i j} \gamma_{i j}+\theta^{2}+\omega_{\alpha} \omega_{\alpha}\right) d V d s- \\
-\left(m_{1}-p_{8}^{2} p_{9}^{2}\right) \int_{0}^{t} \int_{B} \theta,{ }_{i} \theta_{i} d V d s
\end{gathered}
$$

Because the constants $p_{5}, p_{6}, p_{7}, p_{8}$ and $p_{9}$ are arbitrary, we can choose them so that

$$
\begin{gathered}
m_{5} \equiv m_{0}-p_{5}^{2}-p_{6}^{2}>0 m_{6} \equiv m_{0}-\frac{N_{1}^{2}}{p_{5}^{2}}-p_{7}^{2}>0 \\
m_{7} \equiv p_{9}^{2}-\frac{N_{2}^{2}}{\pi_{6}^{2}}-\frac{N_{3}^{2}}{p_{7}^{2}}>0, m_{8} \equiv m_{1}-p_{8}^{2} p_{9}^{2}>0
\end{gathered}
$$


and thus from (34) we are lead to

$$
\begin{aligned}
&\left(m_{2}+m_{4} p_{9}^{2}\right) \int_{0}^{t} \int_{B}\left(\varepsilon_{i j} \varepsilon_{i j}+\gamma_{i j} \gamma_{i j}+\theta^{2}+\omega_{\alpha} \omega_{\alpha}\right) d V d s \geq \\
& \geq m_{0} \int_{B}\left(\dot{u}_{i} \dot{u}_{i}+\dot{\varphi}_{i} \dot{\varphi}_{i}+\theta^{2}\right) d V+m_{5} \int_{B} \varepsilon_{i j} \varepsilon_{i j} d V+ \\
&+m_{6} \int_{B} \gamma_{i j} \gamma_{i j} d V+m_{7} \int_{B} \omega_{\alpha} \omega_{\alpha} d V+m_{8} \int_{B} \theta,{ }_{i} \theta,{ }_{i} d V d s \geq \\
& \quad \geq m_{9} \int_{B}\left(\dot{u}_{i} \dot{u}_{i}+\dot{\varphi}_{i} \dot{\varphi}_{i}+\varepsilon_{i j} \varepsilon_{i j}+\gamma_{i j} \gamma_{i j}+\theta^{2}+\omega_{\alpha} \omega_{\alpha}\right) d V
\end{aligned}
$$

where the semnification of the constant $m_{9}$ is

$$
m_{10}=\min \left\{m_{0}, m_{5}, m_{6}, m_{7}\right\} .
$$

Let us observe that

$$
\begin{array}{r}
\int_{0}^{t} \int_{B}\left(\dot{u}_{i} \dot{u}_{i}+\dot{\varphi}_{i j} \dot{\varphi}_{i j}+\varepsilon_{i j} \varepsilon_{i j}+\gamma_{i j} \gamma_{i j}+\theta^{2}+\omega_{\alpha} \omega_{\alpha}\right) d V d s \geq \\
\geq \int_{0}^{t} \int_{B}\left(\varepsilon_{i j} \varepsilon_{i j}+\gamma_{i j} \gamma_{i j}+\theta^{2}+\omega_{\alpha} \omega_{\alpha}\right) d V d s
\end{array}
$$

Finally, if we choose

$$
m_{3}=\frac{\left(m_{2}+m_{4} p_{9}^{2}\right)}{m_{9}}
$$

then from (35) and (36) we arrive at the desired result (27) and Theorem 3 is proved.

Theorem 1, Theorem 2 and Theorem 3 form the basis of the main result of this study: the uniqueness of solution of mixed initial-boundary value problem for thermoelastic microstretch body with internal state variables.

Theorem 4. Assume that the hypotheses (i) - (v) hold. Then there exists at most one solution of the problem defined by the equations (1), (2) and (6) with the initial conditions (9) and the boundary conditions (10).

Proof. Suppose that the mixed problem has two solutions. Then the difference of these solutions is solution for the above mentioned problem $P_{0}$. For our aim it is suffice to show that the function $y(t)$ defined by

$$
y(t)=\int_{B}\left(\dot{u}_{i} \dot{u}_{i}+\dot{\varphi}_{i j} \dot{\varphi}_{i j}+\varepsilon_{i j} \varepsilon_{i j}+\gamma_{i j} \gamma_{i j}+\theta^{2}+\omega_{\alpha} \omega_{\alpha}\right) d V
$$


vanishes on the interval $\left[0, t_{0}\right]$.

If we assume the contrary, i.e. $y(t) \neq 0$, this is absurdum because the inequality (27) and Gronwall's inequality imply that $y(t) \equiv 0$ on $\left[0, t_{0}\right]$ and Theorem 4 is concluded.

\section{Conclusion.}

The presence of internal state variables do not affect the uniqueness of solution of the mixed problem for microstretch thermoelastic materials.

\section{References}

[1] L. Anand, M.E. Gurtin, A theory of amorphous solids undergoing large deformations Int. J. Solids Struct., Vol. 40(2003), 1465-1487

[2] J.L. Bouvard, D.K. Ward, D. Hossain, E.B. Marin, D.J. Bammann, M.F. Horstemeyer, A general inelastic internal state variable model for amorphous glassy polymers, Acta Mechanica, Vol. 213, 1-2(2010), 71-96

[3] S. Chirita, On the linear theory of thermo-viscoelastic materials with internal state variables, Arch. Mech., Vol. 33(1982), 455-464

[4] A.C. Eringen, Theory of thermo-microstretch elastic solids, Int. J. Engng. Sci., Vol. 28(1990), 1291-1301

[5] C. Flaut, D. Savin, Some examples of division symbol algebras of degree 3 and 5, Carpathian Journal of Mathematics, 31(2): 197- 204, 2015.

[6] G. Groza, M. Jianu, N. Pop, Infinitely differentiable functions represented into Newton interpolating series, Carpathian Journal of Mathematics, 30(3): 309-316, 2014.

[7] L. Harabagiu, O. Simionescu-Panait, Propagation of inhomogeneous plane waves in isotropic solid crystals, Ann. Sci. Univ. Ovidius Constanta, 23 (3): 55-64, 2015

[8] D. Iesan, R. Quintanilla, Some theorems in the theory of microstretch thermopiezo-electricity, Int. J. Engng. Sci., Vol. 45, 1(2007), 1-16

[9] R. Kumar, Wave propagation in a microstretch thermoelastic diffusion solid, Ann. Sci. Univ. Ovidius Constanta, 23 (1): 127-169, 2015.

[10] M. Marin, On existence and uniqueness in thermoelasticity of micropolar bodies, Comptes Rendus, Acad. Sci. Paris, Serie II, vol. 321 (12), 475-480, 1995 
[11] M. Marin, Some basic theorems in elastostatics of micropolar materials with voids, J. Comp. Appl. Math., Vol. 70(1), 115-126, 1996

[12] M. Marin, C. Marinescu, Thermoelasticity of initially stressed bodies. Asymptotic equipartition of energies, Int. J. Eng. Sci., vol. 36 (1), 73-86, 1998

[13] M. Marin, A domain of influence theorem for microstretch elastic materials, Nonlinear Analysis: R.W.A., vol. 11(5), 3446-3452, 2010

[14] M. Marin, Lagrange identity method for microstretch thermoelastic materials, J. Math. Analysis and Applications, vol. 363 (1), pp. 275-286, 2010

[15] K. Sharma, M. Marin, Reflection and transmission of waves from imperfect boundary between two heat conducting micropolar thermoelastic solids, Ann. Sci. Univ. Ovidius Constanta, vol. 22 (2) (2014), 151-175

[16] R.R. Nachlinger, J.W. Nunziato, Wave propagation and uniqueness theorem for elastic materials with ISV, Int. J. Engng. Sci., Vol. 14(1976), $31-38$

[17] J.A. Sherburn , M.F. Horstemeyer, D.J. Bammann, J.R. Baumgardner, Application of the Bammann inelasticity internal state variable constitutive model to geological materials, Geophysical J. Int., Vol. 184, 3(2011), $1023-1036$

[18] K.N. Solanki, D.J. Bammann, A thermodynamic framework for a gradient theory of continuum damage, American Acad. Mech. Conf., New Orleans, 2008

[19] C. Wei, M.M. Dewoolkar, Formulation of capillary hysteresis with internal state variables, Water resources research, Vol. 42(2006), 1-16.

Marin MARIN

Department of Mathematics and Computer Science,

Transilvania University of Brasov,

500091 Brasov, Romania.

Email: m.marin@unitbv.ro

Sorin VLASE

Department of Mechanical Engineering,

Transilvania University of Brasov,

500118 Brasov, Romania

Email: svlase@unitbv.ro 